\title{
Atributos de la organización que aprende: una revisión de la literatura
}

\author{
Esteban López-Zapata \\ Universidad de Antioquia \\ esteban.lopez@udea.edu.co \\ Fernando Enrique García-Muiña \\ Universidad Rey Juan Carlos \\ fernando.muina@urjc.es \\ Susana María García-Moreno \\ Universidad Rey Juan Carlos \\ susanamaria.garcia@urjc.es
}

Resumen: En este artículo se hace una revisión de varios modelos teóricos que han buscado caracterizar a la organización que aprende. A partir de ellos, se ban identifcado coincidencias significativas en atributos como la existencia de una visión compartida, el liderazgo facilitador del aprendizaje, la apertura mental y la experimentación, el pensamiento sistémico, el trabajo en equipo y la comunicación y la integración del conocimiento. Se plantean algunos factores organizativos claves para el desarrollo de estos atributos.

Palabras clave: aprendizaje organizativo, organización que aprende, conocimiento organizativo.

Attributes of the Learning Organization: A review of the literature

Abstract: This article is a review of some theoretical models that have sought to characterize the learning organization, from which we have identified significant overlap in attributes such as the existence of a shared vision, the learning oriented leadership, open-mindedness and experimentation, systems thinking, teamwork and communication, and integration of knowledge. We suggest some organizational factors that would help the development of these attributes.

Key words: organizational learning, learning organization, organizational knowledge. 


\section{Introducción}

Con el avance que ha experimentado la sociedad del conocimiento en las últimas décadas, el aprendizaje organizativo se ha convertido en un campo de interés fundamental para múltiples disciplinas. A partir de la década de 1990 surgen varias corrientes teóricas que buscan explicar la forma como las organizaciones adquieren y gestionan su conocimiento con el fin de mejorar su competitividad, tales como la gestión del conocimiento (Nonaka \& Takeuchi, 1995), el enfoque de la empresa basada en recursos (Barney, 1991), las capacidades dinámicas (Teece, Pisano \& Shuen, 1997), el capital intelectual (Bontis, 1998), la capacidad de absorción (Cohen \& Levinthal, 1990) y la perspectiva del aprendizaje organizativo (Huber, 1991; Crossan, Lane, \& White, 1999).

Estas teorías comparten conceptos similares y coinciden en subrayar la importancia estratégica que tiene el conocimiento para fortalecer la ventaja competitiva de las empresas. Sin embargo, debido a la diversidad de enfoques, no existe un consenso ampliamente aceptado sobre cuáles son los atributos que caracterizan a las empresas que mejor desarrollan su capacidad de aprendizaje, es decir, a las organizaciones que aprenden (the learning organizations).

El objetivo de este artículo es sintetizar los principales atributos que caracterizan a las organizaciones que aprenden e identificar algunos factores sobre los que pueda influir la gerencia para fortalecer la capacidad de aprendizaje organizativo. La compilación y caracterización de estos atributos constituye la principal contribución de este artículo a la literatura sobre el aprendizaje organizativo.

Para lograr este objetivo, se desarrolla un análisis teórico que recoge la evolución conceptual que ha tenido el aprendizaje organizativo y se analiza una serie de propuestas teóricas que han identificado múltiples prácticas, orientaciones y valores corporativos que caracterizan a las organizaciones que aprenden. A partir de estos modelos, se propone un conjunto de dimensiones que sintetizan los atributos planteados por los autores examinados y se propone una serie de recomendaciones que pueden ayudar a fortalecer dichas dimensiones.

\section{El aprendizaje organizativo}

Tradicionalmente, el aprendizaje ha sido un concepto ligado a la psicología para explicar los cambios conductuales y cognitivos que se presentan en las personas. Con el nacimiento de la teoría conductual de la empresa (Cyert \& March, 1963), varios atributos propios de la naturaleza humana han sido considerados como atributos que pueden caracterizar a una organización, y, desde allí, 
diversos autores han considerado que «las organizaciones pueden aprender» $y$ que el aprendizaje organizativo es algo más que la suma de los conocimientos individuales de sus miembros (Hedberg, 1981; Shrivastava, 1983).

En la tabla 1 se presenta una serie de definiciones sobre el concepto de aprendizaje organizativo que ilustran la forma como ha evolucionado este campo del conocimiento.

Las primeras definiciones tienen una marcada influencia del enfoque conductual y se centran principalmente en la forma en que las organizaciones adaptan su comportamiento a las variaciones del entorno. Desde este enfoque, se considera que una organización aprende cuando se evidencia un cambio en sus acciones (e.g. Cyert \& March, 1963). Posteriormente, surgen definiciones más relacionadas con los cambios cognitivos que ocurren en la organización cuando esta adquiere nueva información, de tal manera que se empieza a considerar que una organización aprende cuando se evidencia un cambio en sus conocimientos, aunque ello no implique un cambio en sus acciones (e.g. Duncan \& Weiss, 1979). Finalmente, aparecen definiciones un poco más amplias que no solo consideran los cambios cognitivos y conductuales, sino que incluyen los múltiples niveles en los que ocurre el aprendizaje (individual, grupal, organizativo) y los múltiples procesos que constituyen dicho fenómeno (e.g. Huber, 1991; Crossan et alii, 1999).

Teniendo en cuenta esta evolución y la relevancia de los enfoques más actuales, se propone definir el aprendizaje organizativo como una capacidad dinámica de la organización que involucra la exploración de nuevo conocimiento y la explotación de su conocimiento actual, a través de procesos de adquisición de conocimiento, distribución, interpretación y memoria organizativa, con el fin de modificar su estructura cognitiva e influir positivamente sobre el cambio organizativo y los resultados empresariales.

De esta manera, se reconocen varios atributos claves del concepto de aprendi$z a j e$ que han adquirido relevancia en los enfoques más actuales: su identificación como capacidad dinámica que le permite integrar, construir y reconfigurar las rutinas organizativas para enfrentar el cambio (Teece, 2009), su carácter ambidiestro que involucra el desarrollo simultáneo de la exploración y explotación del conocimiento (March, 1991; Raisch \& Birkinshaw, 2008; López-Zapata, García-Muiña \& García-Moreno, 2012) y su carácter multidimensional que involucra los procesos que intervienen desde la adquisición del conocimiento hasta su almacenamiento o institucionalización en la memoria organizativa (Huber, 1991; Crossan et alii, 1999). 


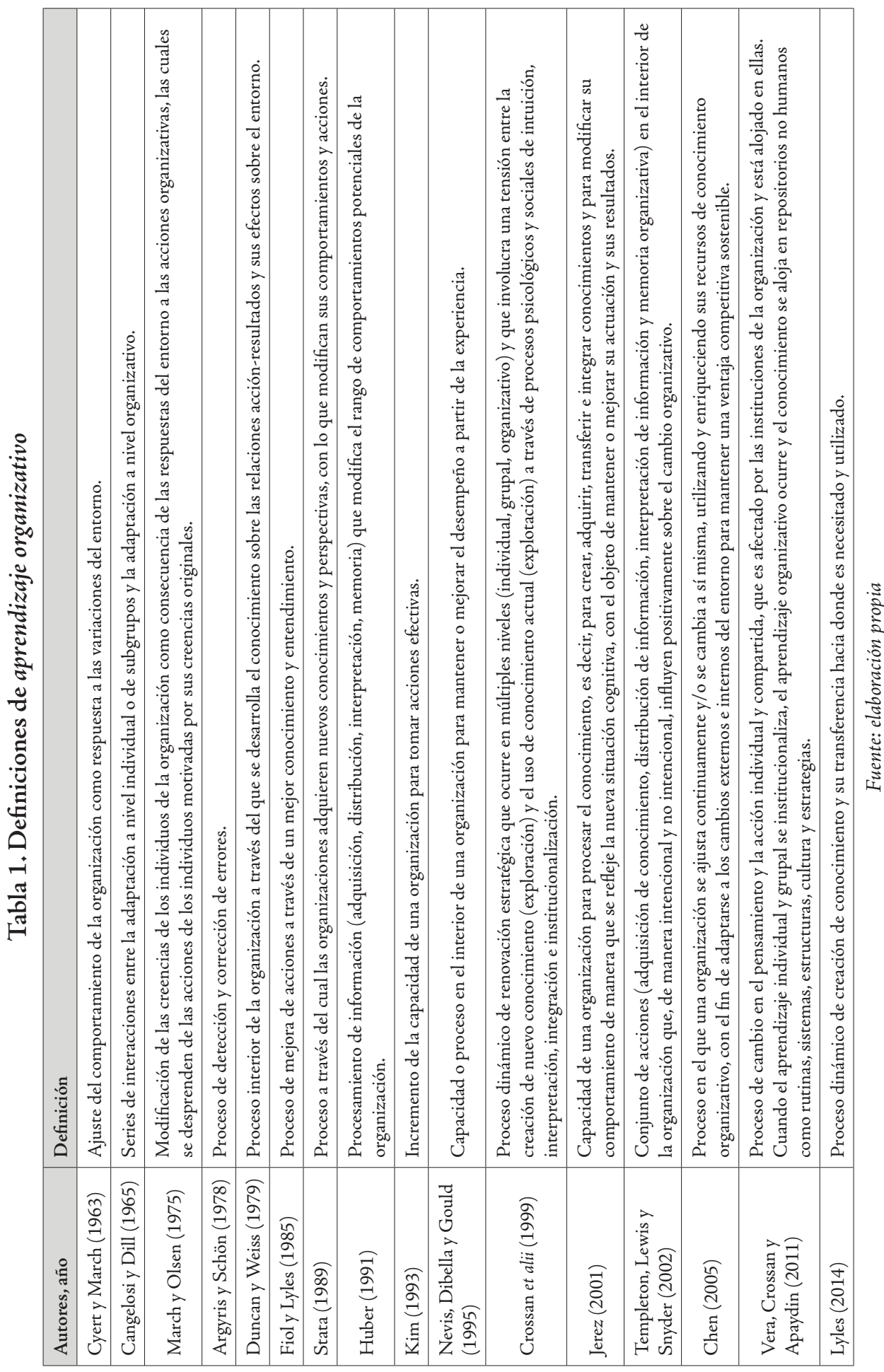




\section{La organización que aprende}

De forma paralela al desarrollo de los modelos de aprendizaje organizativo que buscan responder a la pregunta ¿cómo aprenden las organizaciones?, surgió otro enfoque teórico que busca responder a la pregunta ¿qué caracteriza a las organizaciones que aprenden mejor? Dicho enfoque se conoce como la organización que aprende.

El concepto de la organización que aprende se popularizó a raíz de la publicación de La quinta disciplina (Senge, 1990), que propone cinco disciplinas que caracterizan a las empresas abiertas al aprendizaje. Dichas disciplinas son el dominio personal, los modelos mentales, la construcción de una visión compartida, el aprendizaje en equipo y el pensamiento sistémico. En términos generales, la organización que aprende es una organización que es competente en la creación, adquisición y transferencia de conocimiento y en la modificación de su comportamiento para reflejar el nuevo conocimiento (Garvin, 1993, p. 80).

Se destaca entonces que ambas corrientes teóricas se refieren a un mismo fenómeno de estudio, aunque existen algunas diferencias en sus objetivos y métodos (Easterby-Smith, 1997; Easterby-Smith, Crossan, \& Nicolini, 2000; Shipton, 2006). En la tabla 2 se presenta un paralelo entre ambos enfoques de acuerdo con un análisis desarrollado por Tsang (1997).

Tabla 2. Aprendizaje organizativo vs, la organización que aprende

\begin{tabular}{|c|c|c|}
\hline Criterio de comparación & Aprendizaje organizativo & La organización que aprende \\
\hline Enfoque & Descriptivo & Prescriptivo \\
\hline Pregunta clave & $\begin{array}{l}\text { ¿Cómo aprende una } \\
\text { organización? }\end{array}$ & $\begin{array}{l}\text { ¿Cómo debería aprender una } \\
\text { organización? }\end{array}$ \\
\hline Audiencia objetivo & Académicos & Profesionales \\
\hline Objetivo & Construir teoría & $\begin{array}{l}\text { Mejorar el desempeño } \\
\text { organizativo }\end{array}$ \\
\hline Fuentes de información & $\begin{array}{l}\text { Recolección sistemática de } \\
\text { datos }\end{array}$ & Experiencias de consultoría \\
\hline Metodología & $\begin{array}{l}\text { Métodos de investigación } \\
\text { rigurosos }\end{array}$ & $\begin{array}{l}\text { Estudios de casos laxos e } \\
\text { investigación-acción }\end{array}$ \\
\hline Generalización & $\begin{array}{l}\text { Atención a los factores que } \\
\text { limitan la generalización de los } \\
\text { resultados de investigación }\end{array}$ & $\begin{array}{l}\text { Tendencia a la generalización } \\
\text { excesiva de la teoría para todos } \\
\text { los tipos de organización }\end{array}$ \\
\hline Resultado del aprendizaje & $\begin{array}{l}\text { Cambios potenciales del } \\
\text { comportamiento }\end{array}$ & $\begin{array}{l}\text { Cambios reales del } \\
\text { comportamiento }\end{array}$ \\
\hline $\begin{array}{l}\text { Relación entre } \\
\text { aprendizaje y desempeño }\end{array}$ & $\begin{array}{l}\text { Se reconoce que puede ser } \\
\text { positiva o negativa }\end{array}$ & Se asume que es positiva \\
\hline
\end{tabular}

Fuente: Tsang (1997) 
Por una parte, la corriente del aprendizaje organizativo tiene un marcado enfoque descriptivo, busca construir teoría a partir de métodos rigurosos y su público de interés son las comunidades académicas. Por otra parte, la corriente de la organización que aprende tiene un marcado enfoque prescriptivo, busca crear técnicas y recomendaciones prácticas para mejorar el desempeño organizativo, a partir de casos de estudio y experiencias de consultoría, y su público de interés son los profesionales, los consultores y las empresas.

Muchos académicos han sido críticos con la literatura prescriptiva de la organización que aprende, ya que varios de sus planteamientos provienen de consultores que sintetizan sus experiencias, sin tener una metodología de investigación rigurosa que sustente la generalización de sus ideas a diferentes tipos de empresas, culturas y entornos. Por otra parte, muchos empresarios y consultores han sido críticos con la corriente academicista del aprendizaje organizativo, ya que muchas investigaciones de este enfoque presentan resultados poco relevantes o difíciles de interpretar para mejorar el desempeño. Tsang (1997) considera que esta dicotomía no debería existir, sino que ambos enfoques podrían complementarse para construir teorías que sean, a su vez, rigurosas científicamente, pero relevantes desde el punto de vista empresarial.

A partir de este enfoque, surgen múltiples propuestas teóricas de atributos, prácticas y valores que caracterizan a la organización que aprende, algunas de las cuales han intentado combinar el rigor y la relevancia de los enfoques descriptivo y prescriptivo. En la tabla 3 se presenta un resumen de los atributos que diversos autores han identificado en este tipo de organizaciones.

Se puede observar que existen algunos atributos que aparecen de forma reiterada, como la existencia de una visión compartida, el liderazgo facilitador o el compromiso de los directivos con el aprendizaje, la apertura mental y la experimentación, el pensamiento sistémico, el trabajo en equipo, la comunicación o el diálogo y la integración del conocimiento. A continuación se presenta una descripción de cada uno de estos atributos o dimensiones que caracterizan a la organización que aprende, tomando como base el análisis comparativo que hemos desarrollado de estos diversos modelos teóricos. 


\begin{tabular}{|c|c|c|c|c|c|c|c|c|c|}
\hline \multirow{6}{*}{ 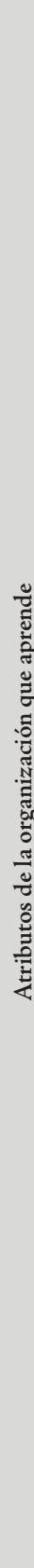 } & 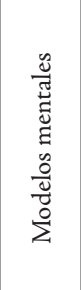 & & & & & 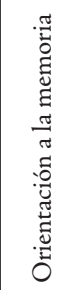 & & 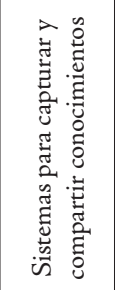 & 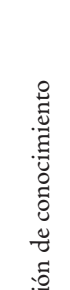 \\
\hline & 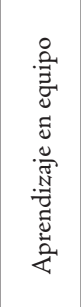 & 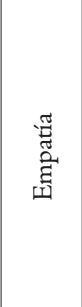 & 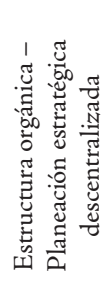 & 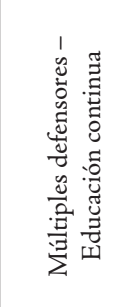 & 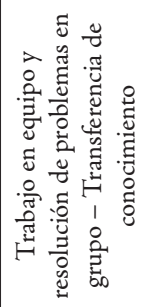 & 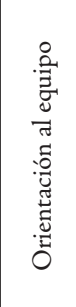 & & 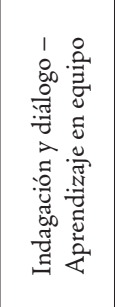 & 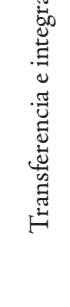 \\
\hline & 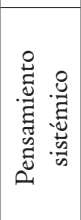 & 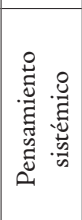 & & 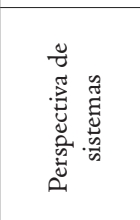 & & 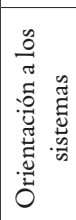 & & 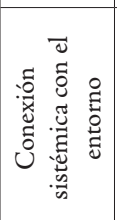 & 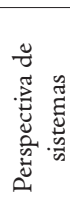 \\
\hline & & 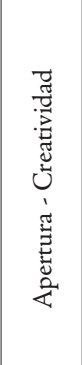 & 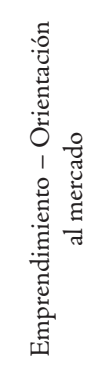 & 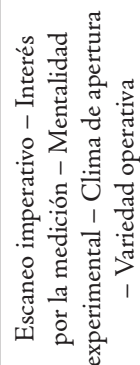 & 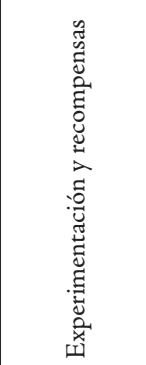 & \multirow{2}{*}{ 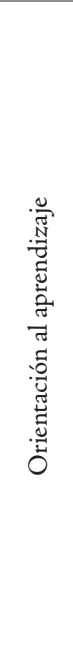 } & 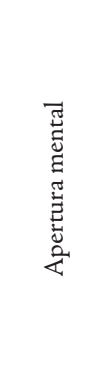 & 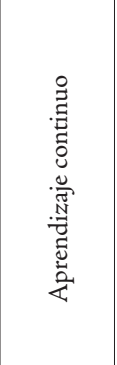 & 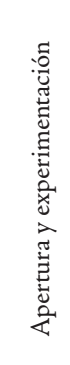 \\
\hline & 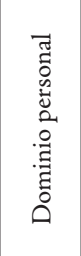 & 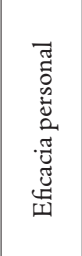 & 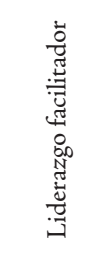 & 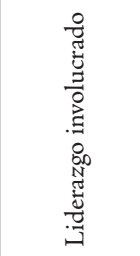 & 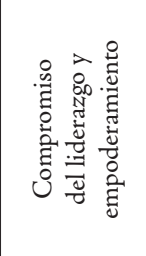 & & 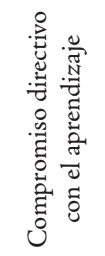 & 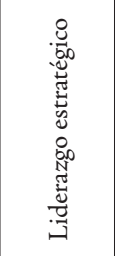 & 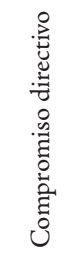 \\
\hline & 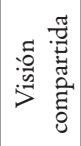 & & & 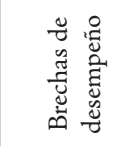 & 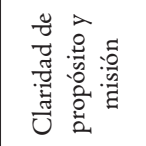 & & 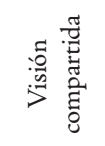 & 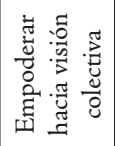 & \\
\hline 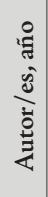 & 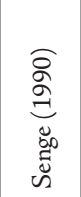 & 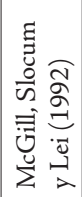 & 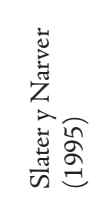 & 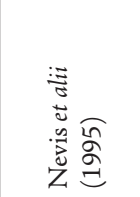 & 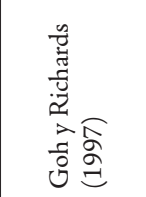 & 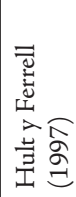 & 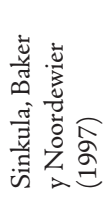 & 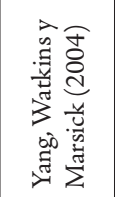 & 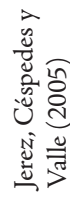 \\
\hline
\end{tabular}


Esteban López-Zapata, Fernando Enrique García-Muiña, Susana María García-Moreno

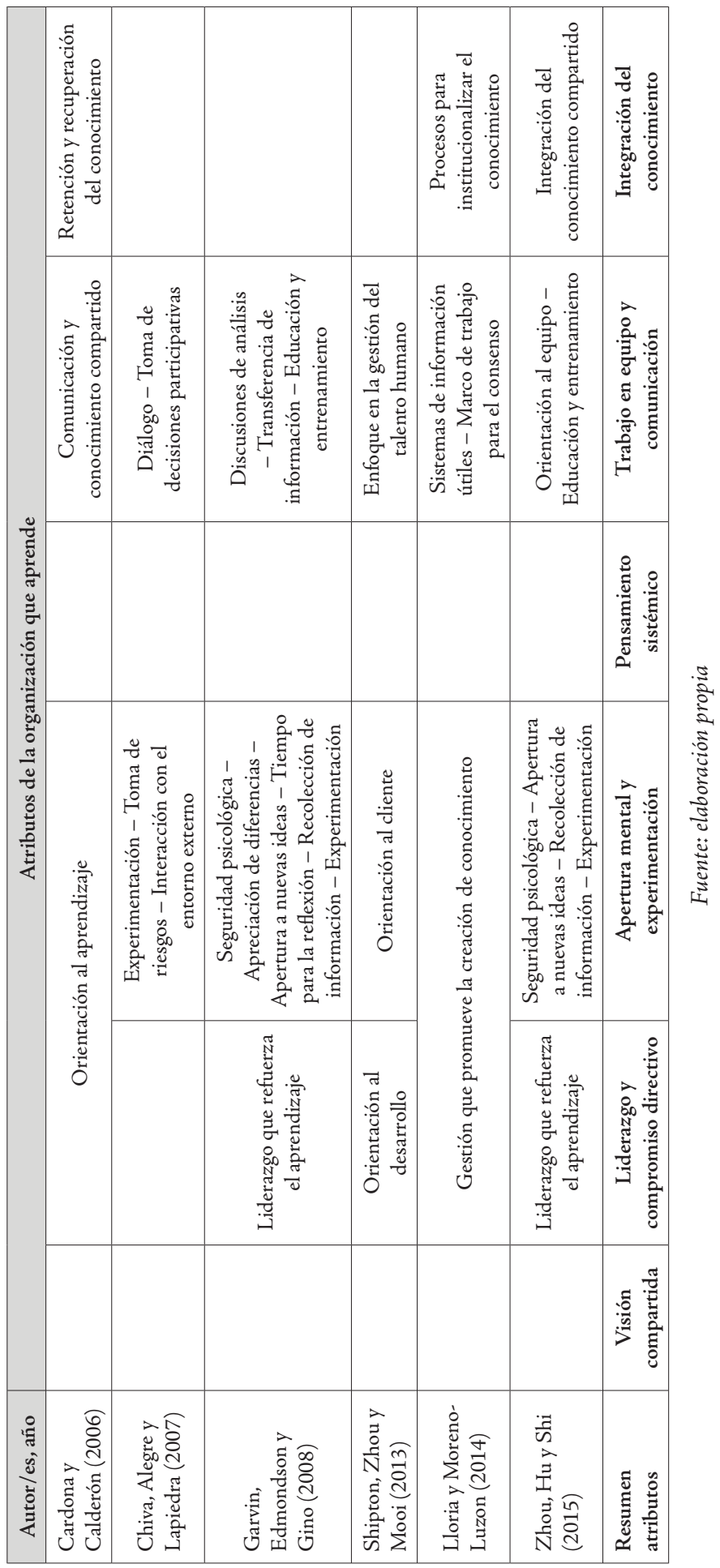




\subsection{Visión compartida}

La visión compartida es una condición fundamental de la organización que aprende, ya que brinda una dirección que permite enfocar las energías y propósitos de todos sus miembros hacia un aprendizaje proactivo (Sinkula et alii, 1997). Al visibilizar un estado futuro deseado, se crea una tensión que promueve el aprendizaje, orientando a la organización hacia dicho futuro deseado (Goh \& Richards, 1997). La visión compartida ayuda a los miembros de la organización a considerar el valor potencial de intercambiar su conocimiento y facilita el logro de consensos en relación con las metas comunes, con lo que se facilita la interpretación compartida del conocimiento.

La visión ha sido un concepto frecuentemente abordado en la literatura sobre liderazgo empresarial, pero en muchos casos las empresas asumen el concepto como una imposición de una persona (líder) o de un grupo particular (equipo directivo), de tal manera que entre los miembros de la organización se genera un acatamiento a la visión, pero no un compromiso. Para que una visión sea realmente compartida, es necesario que despierte el compromiso de todos los miembros de la empresa, para lo que esta debe reflejar los intereses y visiones particulares de los individuos. Las visiones compartidas derivan su fuerza del interés común y del deseo de las personas de estar conectadas a una tarea que consideran importante (Senge, 1990). Contraria al enfoque tradicional de arriba a abajo, la visión compartida se puede construir a través del involucramiento activo de todos los miembros de la empresa en el desarrollo, la comunicación y la implementación de los objetivos corporativos, que hace que las personas jueguen un rol activo en la creación de su propio norte (Wang \& Rafiq, 2009).

Igualmente, para generar verdadera motivación, la visión compartida debe ser retadora y plantear un estado deseado mucho mejor que el estado actual. La percepción de que existe una brecha entre el desempeño real y el desempeño deseado es importante porque impulsa a la organización a reconocer su necesidad de aprender y mejorar (Nevis et alii, 1995).

Teniendo en cuenta estas condiciones, las decisiones estratégicas y las competencias de los líderes del equipo directivo juegan un rol clave en la construcción de una visión que realmente sea compartida por toda la organización. Es fundamental que el equipo directivo tenga la sensibilidad suficiente para percibir los intereses particulares de todos los miembros de la empresa, de tal forma que las decisiones estratégicas reflejen dichos intereses. 


\subsection{Liderazgo y compromiso directivo}

En consonancia con el concepto planteado de la visión compartida, se hace necesaria la existencia de un liderazgo que facilite la construcción de dicha visión y que esté comprometido con el aprendizaje como un proceso colectivo. El compromiso directivo se manifiesta cuando el aprendizaje es asumido como un factor estratégico de la empresa que determina sus resultados a largo plazo, de tal forma que los empleados entiendan su importancia y se involucren en su consecución (Hult \& Ferrell, 1997; Jerez et alii, 2005; Cardona \& Calderón, 2006).

El liderazgo es mencionado reiteradamente como un elemento que promueve un clima de aprendizaje a través de conductas como la búsqueda de realimentación, la apertura hacia la crítica, la aceptación de errores y el empoderamiento de los empleados para que tomen decisiones y asuman riesgos (Garvin, 1993; Slocum, McGill, \& Lei, 1994; Nevis et alii, 1995; Goh \& Richards, 1997). El comportamiento de las personas refleja el comportamiento de los líderes. De este modo, si los líderes otorgan una alta prioridad a la identificación de problemas, la transferencia de conocimiento y las reflexiones posteriores al desarrollo de un proyecto, es más probable que este tipo de acciones se desarrollen con naturalidad. Cuando los líderes demuestran la voluntad de considerar múltiples puntos de vista, los empleados se atreven a ofrecer nuevas ideas y visiones alternativas (Garvin et alii, 2008).

El líder facilitador del aprendizaje se caracteriza porque se orienta al desarrollo de las personas que lo rodean, genera un ambiente que motiva a las personas a querer aprender más, pero, en lugar de asumir el rol de experto que enseña a sus subordinados «cómo se hacen las cosas», asume el rol de entrenador (coach) que ayuda a sus subordinados a descubrir por sí mismos los patrones y suposiciones que subyacen en la realidad organizativa y a asumir la responsabilidad de su propio aprendizaje para tomar mejores decisiones (Slater \& Narver, 1995). Este estilo de liderazgo suele incluir los comportamientos propios del liderazgo transformacional: el carisma, la consideración individualizada, la motivación inspiracional y la estimulación intelectual (Bass \& Riggio, 2006; Zhou et alii, 2015)

Una condición fundamental para desarrollar este estilo de liderazgo es la eficacia personal. Los directivos con un fuerte sentido de eficacia personal sienten que pueden aprender a influir significativamente sobre su entorno, dichos directivos se caracterizan por un alto nivel de autoconsciencia, dominio personal y una alta proactividad en la resolución de problemas (Senge, 1990; McGill et alii, 1992). 
Claramente, el desarrollo generalizado de estos estilos de liderazgo depende de la forma como esté conformado el equipo directivo de la organización. No es probable que los jefes de niveles intermedios desarrollen un liderazgo orientado al aprendizaje cuando dicho estilo no es evidente en los líderes del equipo directivo. Un reto contemporáneo que enfrentan las áreas de gestión humana es desarrollar prácticas innovadoras que promuevan estos estilos de liderazgo en todos los niveles de la organización.

\subsection{Apertura mental y experimentación}

Cuando las empresas cuestionan de forma proactiva sus rutinas, suposiciones y creencias sostenidas por largo tiempo, están dando un primer paso para el aprendizaje y la transformación organizativa (Hedberg, 1981; Sinkula et alii, 1997). Gran parte del aprendizaje surge de la interacción cotidiana de las personas, para lo que se requiere un clima de apertura que permita a las personas expresar libremente sus puntos de vista (Nevis et alii, 1995).

Para crear un clima de apertura es importante fomentar la diversidad cultural y funcional, generando una disposición de las personas a aceptar diferentes opiniones y experiencias, evitando la actitud de prejuzgar las ideas ajenas como si fueran inferiores a las propias (Nevis et alii, 1995; Jerez et alii, 2005). Es importante que las personas desarrollen una humildad funcional que les permita considerar que sus propios valores y creencias no son necesariamente mejores o peores que los valores y creencias de los demás (McGill et alii, 1992). Algunas condiciones que favorecen este clima de apertura son la seguridad psicológica de los empleados para expresar sus pensamientos, la apreciación de las diferencias, la aceptación de nuevas ideas, la dedicación de tiempo para la reflexión, la recolección de información del entorno y la experimentación (Garvin et alii, 2008).

La experimentación se refiere a la búsqueda sistemática y ensayo de nuevos conocimientos, para lo cual es esencial el uso del método científico. Esto implica la búsqueda de soluciones flexibles e innovadoras para problemas actuales y futuros, a partir del uso de diferentes métodos y procedimientos (Garvin, 1993; Jerez et alii, 2005). A medida que los directivos, además de desempeñar su rol tradicional como gerentes, son capaces de actuar como investigadores aplicados y ver cada etapa de la cadena de valor no solo como una actividad productiva, sino también como un experimento de aprendizaje, aumenta la posibilidad de que la organización desarrolle aprendizajes realmente valiosos (Nevis et alii, 1995). La importancia que brindan las organizaciones a la experimentación puede evidenciarse en sus esfuerzos de investigación y desarrollo. 
Tanto la apertura mental como la experimentación están asociadas con otros valores y competencias como la creatividad, el emprendimiento y la tolerancia al riesgo. La creatividad es una competencia vital para impulsar el aprendizaje y, tal vez, una de las más difíciles de desarrollar, ya que implica cierta tolerancia a la ambigüedad y la flexibilidad personal para adaptar el propio comportamiento a circunstancias cambiantes (McGill et alii, 1992). El emprendimiento, que se evidencia en el aprovechamiento de nuevas oportunidades de negocio a través de la innovación, implica la existencia de una cultura que favorece la exploración, el cuestionamiento de las creencias arraigadas y el desarrollo de nuevos comportamientos (Slater \& Narver, 1995). Igualmente, la tolerancia al riesgo es una condición crítica, pero, a pesar de su importancia, las sanciones asociadas al fracaso hacen que muchas personas prefieran tomar pocos riesgos, con lo que se desincentiva el aprendizaje. Para promover la experimentación es importante que la estructura y los sistemas estén diseñados para apoyar esta práctica, impulsando cierta libertad para probar nuevos métodos de trabajo y procesos innovadores. Los sistemas de compensación, por ejemplo, deberían recompensar la innovación y la disposición a asumir riesgos (Goh \& Richards, 1997).

Otra característica asociada a la apertura mental y la experimentación es la interacción de la organización con el entorno externo (Nevis et alii, 1995; Chiva et alii, 2007; Garvin et alii, 2008) y, en particular, la orientación al mercado (Slater \& Narver, 1995; Shipton et alii, 2013), que implica dar la más alta prioridad a la creación de valor para los clientes y su mantenimiento de forma rentable, a partir de la generación de inteligencia de mercados sobre clientes, competidores y otros grupos de interés.

Se observa que la apertura mental y la experimentación son atributos de la cultura corporativa que pueden desarrollarse a largo plazo a través del planteamiento de estrategias empresariales y prácticas de gestión humana que incentiven dichas condiciones. La decisión de incursionar en un nuevo mercado o la implementación de un sistema de recompensas para ideas innovadoras son ejemplos de decisiones encaminadas en esta dirección.

\subsection{Pensamiento sistémico}

El pensamiento sistémico está relacionado con la capacidad de identificar las conexiones entre diversos asuntos, eventos o datos y percibir el todo como algo más que la suma de sus partes (McGill et alii, 1992). Esto implica identificar la interdependencia de las unidades organizativas, considerar los problemas y soluciones en términos de las relaciones sistémicas entre procesos y conectar los objetivos de las unidades con los objetivos de la organización (Nevis et alii, 1995; Yang et alii, 2004). 
El surgimiento de la dinámica de sistemas como herramienta esencial para analizar en profundidad todo tipo de sistemas sociales, que se caracterizan por su alta complejidad, es uno de los paradigmas centrales que fundamentó la literatura precursora de la organización que aprende (Forrester, 1971; Senge, 1990). La dinámica de sistemas permitió entender las organizaciones como enormes redes de nodos interconectados donde los cambios generados en un nodo producen efectos, positivos o negativos, en los demás nodos, de tal forma que una organización que aprende requiere tener una visión holística de sí misma para tomar mejores decisiones y considerar las múltiples conexiones que existen entre sus unidades o entre la misma empresa con su entorno externo (Stata, 1989). Concebir una organización como un sistema complejo interconectado requiere que los directivos sean conscientes de los flujos de información y de poder que subyacen en esta, y que sean capaces de identificar los desacuerdos legítimos que surgen entre diferentes unidades, enfocando las energías de todos a comprender la esencia de dichos desacuerdos por encima de los conflictos políticos.

Al igual que la visión compartida, el pensamiento sistémico impulsa a los miembros de la organización a mantener una identidad común y permite que los diferentes grupos o individuos tengan una idea clara de los objetivos organizativos, entendiendo la forma particular como cada uno de ellos ayuda a alcanzarlos (Hult \& Ferrell, 1997; Jerez et alii, 2005).

Las decisiones asociadas al diseño de la estructura organizativa con un soporte adecuado de tecnologías de la información pueden ser factores claves en el desarrollo del pensamiento sistémico dentro de la empresa. En la medida en que la estructura y las tecnologías favorecen los vínculos y el intercambio de información entre los múltiples procesos organizativos es más probable que los individuos responsables de cada proceso desarrollen una visión más integral de todos los procesos de la empresa.

\subsection{Trabajo en equipo y comunicación}

Para que el aprendizaje colectivo sea algo más que la suma de los aprendizajes de los individuos, es fundamental que la cultura corporativa favorezca el trabajo en equipo y la comunicación, valores claves para potenciar la asimilación del conocimiento en el interior de la organización.

El trabajo en equipo permite que el conocimiento sea compartido entre los individuos y que cada uno tenga una mejor comprensión de las necesidades de los demás. El aprendizaje se potencia cuando las estructuras y sistemas de la organización promueven la solución de problemas en equipo, que tengan la capacidad de interactuar de manera transversal con las diferentes áreas funcionales para 
favorecer la transferencia de conocimiento entre estas (Goh \& Richards, 1997). El aprendizaje en equipo es un proceso que involucra la alineación y el desarrollo de competencias colectivas que surgen con la experiencia. Cuando un equipo no está alineado hacia objetivos comunes, se genera un alto desperdicio de energía; los individuos pueden poner mucho empeño, pero sus esfuerzos no se traducen en un mejor desempeño colectivo. Sin embargo, cuando el equipo está alineado hacia una dirección común, las energías individuales se armonizan y se genera una sinergia que se manifiesta en un desempeño superior de todo el equipo como conjunto (Senge, 1990).

Una cultura que favorece el trabajo en equipo se caracteriza también por la participación de los individuos en la toma de decisiones. Las empresas que asumen la planeación estratégica bajo un enfoque descentralizado, que prioriza la participación de los individuos y los equipos en las decisiones estratégicas, son organizaciones más abiertas al aprendizaje (Slater \& Narver, 1995). Para que la participación sea efectiva, es indispensable que los empleados tengan acceso a la información relevante que implica cada decisión (Chiva et alii, 2007).

En este mismo sentido, la comunicación efectiva es esencial para favorecer la transferencia de conocimiento y la generación de interpretaciones comunes. En la medida en que se promueve la comunicación y la empatía, se genera confianza, respeto por la dignidad de las personas, comportamiento ético, voluntad de tomar la responsabilidad de las relaciones y compromiso para solucionar los conflictos (McGill et alii, 1992; Cardona \& Calderón, 2006).

Existen dos estilos discursivos claves que pueden orientar la comunicación en un equipo: la discusión y el diálogo (Senge, 1990). La discusión se caracteriza por que los individuos presentan y defienden distintos puntos de vista, buscan analizar situaciones, valorar cada perspectiva para elegir la mejor, llegar a acuerdos y tomar decisiones. Por otra parte, el diálogo se caracteriza por su estilo divergente, no se procura llegar a un acuerdo, sino lograr comprensiones más amplias y matizadas de asuntos complejos, se presentan distintos puntos de vista, no para elegir el mejor, sino con la mente abierta para generar nuevos puntos de vista que ninguna de las personas habría considerado individualmente antes de iniciar la conversación; en un diálogo, el objetivo no es convencer al otro, sino generar mejores interpretaciones colectivas sobre el asunto dialogado. El diálogo es entonces una indagación colectiva sostenida sobre los procesos, suposiciones y certezas que constituyen la experiencia cotidiana, es un proceso básico para construir interpretaciones comunes, ya que permite dilucidar los significados ocultos de las palabras (Chiva et alii, 2007). 
El aprendizaje en los equipos de trabajo depende significativamente de su capacidad para equilibrar adecuadamente sus prácticas de diálogo y de discusión. Ambos tipos de comunicación son importantes, pero las reglas de juego son diferentes, las metas son diferentes y, si los equipos no saben distinguirlas, no tendrán ni diálogos ni discusiones productivas (Senge, 1990; Hult \& Ferrell, 1997). El diálogo auténtico no es el que busca llegar a un consenso, sino promover el pluralismo de perspectivas, lo que enriquece el aprendizaje y permite confrontar los modelos mentales que subyacen en la cultura corporativa (Argyris \& Schön, 1978; Easterby-Smith et alii, 2000). Pero la discusión también es necesaria para tomar decisiones que permitan resolver los problemas cotidianos de la organización, fortalecer la visión compartida y dirimir los conflictos de manera constructiva y racional (Senge, 1990; Hult \& Ferrell, 1997; Garvin et alii, 2008)

Además de la comunicación interna que se presenta entre los miembros de un equipo, es de suma importancia la comunicación externa que se presenta entre múltiples equipos para convertir el conocimiento grupal en conocimiento organizativo. La transferencia de conocimiento debe ser transversal a las diversas áreas funcionales, superando las barreras que suelen formarse entre las unidades organizativas y entre la empresa y los grupos de interés externos (Goh \& Richards, 1997; Jerez et alii, 2005; Garvin et alii, 2008). Para fomentar estos flujos de conocimiento es esencial contar con una estructura orgánica, que se distingue por su fluida y extensiva comunicación lateral, la descripción flexible de las responsabilidades de cada cargo, el reconocimiento de interdependencia que motiva a los individuos a cooperar y la existencia de mecanismos de enlace, tales como comités, roles integradores o estructuras matriciales (Mintzberg, 1988; Gupta \& Govindarajan, 1991; Slater \& Narver, 1995).

Otro elemento formal que potencializa el trabajo en equipo y la comunicación, generando una mejor asimilación del conocimiento, es la educación continua y el entrenamiento. En la medida en que las empresas se comprometen con estrategias de formación y entrenamiento a lo largo de todas sus unidades, la transferencia de conocimiento se convierte en un proceso natural para sus miembros. Es importante desarrollar programas formales e informales de educación, tanto para los nuevos empleados contratados, como para los empleados que adquieren nuevos roles y para todos los miembros de forma periódica para apoyar procesos de transformación organizativa y mantener actualizados los conocimientos específicos de la industria (Nevis et alii, 1995; Garvin et alii, 2008).

De esta manera, se observa que las decisiones asociadas a la estructura organizativa, las políticas de gestión humana y las tecnologías de la información son fundamentales en el desarrollo de una cultura corporativa que privilegie el trabajo en equipo y la comunicación. 


\subsection{Integración del conocimiento}

Finalmente, la integración del conocimiento está relacionada con la capacidad que tiene la empresa para almacenar el conocimiento colectivo que ha ido construyendo con el tiempo y poderlo recuperar cuando sea requerido. Implica la creación de un cuerpo colectivo de conocimiento que permanezca en la empresa y pueda ser utilizado en futuras situaciones, a pesar de la rotación natural de los miembros de la empresa (Jerez et alii, 2005). Puede observarse que este atributo está netamente ligado al concepto de memoria organizativa (Huber, 1991; Hult \& Ferrell, 1997), que reside tanto en la mente de los individuos como en la cultura corporativa, los espacios de trabajo, las rutinas organizativas, las estructuras, los sistemas de información y los archivos externos (Walsh \& Ungson, 1991).

Una parte esencial del conocimiento que poseen las organizaciones se encuentra alojada en los modelos mentales de sus individuos (Senge, 1990). Los modelos mentales representan la forma como una persona ve el mundo - incluyendo sus comprensiones implícitas y explícitas-, forman un contexto a través del que se analiza e interpreta la nueva información que se recibe y ayudan a determinar la relevancia del conocimiento almacenado para ser usado en nuevas situaciones (Kim, 1993). La integración del conocimiento se fortalece cuando los individuos socializan sus modelos mentales individuales $y$, de esta manera, construyen modelos mentales compartidos que se institucionalizan a través de la cultura y sus diversos elementos (símbolos, ritos, lenguaje, historias y valores compartidos).

El conocimiento colectivo también se integra a través de las rutinas organizativas, que son para las empresas un concepto equivalente a lo que las habilidades son para un individuo: una forma de conocimiento que se desarrolla con la práctica y que está necesariamente codificado de forma explícita (Nelson \& Winter, 1982). El conocimiento tácito que reposa en las rutinas organizativas es de alto valor estratégico, ya que es mucho más difícil de imitar que el conocimiento explícito (Kogut \& Zander, 1992; Zollo \& Winter, 2002; Nonaka \& Von Krogh, 2009).

Aunque se destaca el valor del conocimiento tácito, no se debe desconocer la importancia del conocimiento explícito que reposa en elementos más tangibles, como las estructuras, los documentos y los sistemas de información. Uno de los mayores retos del aprendizaje organizativo es el alto coste que implica grabar el conocimiento que se adquiere, almacenarlo y hacerlo fácil de recuperar cuando se necesita (Levitt \& March, 1988). Por esta razón, las tecnologías de la información desempeñan un rol central en la integración del conocimiento y su institucionalización (Lloria \& Moreno-Luzon, 2014). 
La memoria organizativa involucra dos funciones claves: el almacenamiento y la recuperación del conocimiento. Aunque el almacenamiento del conocimiento ocurre a través de agentes humanos y no humanos, en la recuperación del conocimiento la participación de las personas es indispensable, ya que es la inteligencia humana la que permite articular experiencias e integrarlas con los elementos no humanos como los sistemas de información (Bontis, Crossan, 8 Hulland, 2002; Cardona \& Calderón, 2006).

\section{Conclusiones}

La evolución que ha tenido la literatura del aprendizaje organizativo durante las últimas décadas y la popularización del enfoque de la organización que aprende ha promovido el surgimiento de múltiples modelos teóricos que identifican los atributos de las empresas con mayor capacidad de aprendizaje.

Una revisión de estos modelos ha permitido identificar seis dimensiones generales que agrupan los atributos de la organización que aprende: (1) la existencia de una visión compartida, (2) el liderazgo facilitador del aprendizaje, (3) la apertura mental y la experimentación, (4) el pensamiento sistémico, (5) el trabajo en equipo y la comunicación y (6) la integración del conocimiento. La identificación y caracterización de estas dimensiones constituye el principal aporte de este artículo al estado de la cuestión sobre la organización que aprende. A partir de esta revisión, se identifican varios factores que la empresa podría intervenir para favorecer su desarrollo, tales como la estrategia, la estructura, las políticas de gestión humana, las tecnologías de la información y la composición del equipo directivo.

En primer lugar, las decisiones estratégicas pueden ayudar a definir la capacidad de aprendizaje a largo plazo, ya que ayudan a definir la visión compartida de la empresa y generan incentivos que bien pueden fomentar el aprendizaje o desestimularlo. Autoras como Fiol y Lyles (1985) ya intuían que la estrategia y la estructura eran antecedentes claves de la capacidad de aprendizaje. Por ejemplo, una empresa que decide desarrollar nuevos productos, diversificar sus negocios o ingresar a nuevos mercados deberá promover un clima de apertura y experimentación que facilite la ejecución de dichas estrategias y necesitará promover la comunicación e integración del conocimiento que permitan aprovechar sus conocimientos actuales en los nuevos negocios y mercados. Por otra parte, las estrategias que implican que la empresa se mantenga en su zona de confort (mismos productos, mismos clientes, mismas tecnologías) son decisiones que a largo plazo desincentivan el aprendizaje. 
En segundo lugar, las decisiones asociadas a la estructura organizativa también influyen sobre el aprendizaje. Una estructura orgánica y descentralizada favorece más el trabajo en equipo y la comunicación que una estructura mecanicista y centralizada. El uso de mecanismos de enlace como comités permanentes, directivos integradores y estructuras matriciales puede ser muy útil para favorecer los flujos de información y la toma de decisiones participativas, generando así un entorno más propicio para la transferencia interna del conocimiento y el pensamiento sistémico.

En tercer lugar, las políticas de gestión humana repercuten directamente sobre los atributos de la organización que aprende. Las prácticas de gestión humana suelen ser una de las principales herramientas para desarrollar la capacidad de aprendizaje de una empresa (Jerez, 2001). Por ejemplo, el diseño sistémico de los procesos alrededor de equipos de personas más que de individuos, el reclutamiento de individuos polivalentes que se ajusten a una cultura de apertura mental y experimentación, la inversión intensiva en programas de formación y la compensación variable que recompense los resultados colectivos de los equipos, la creatividad, la flexibilidad, el emprendimiento y la tolerancia al riesgo son políticas que podrían fortalecer la cultura de una organización que aprende.

En cuarto lugar, las tecnologías de la información pueden ser un apoyo valioso para el aprendizaje. Autores como Alavi y Leidner (2001) han explorado el rol que desempeñan las TIC en la gestión del conocimiento. Se ha mencionado la importancia de estas herramientas para la integración de conocimiento y el aprovechamiento de la memoria organizativa, pero también pueden ser un apoyo para la adquisición de conocimiento externo, ya que favorecen la apertura mental, pueden fomentar el pensamiento sistémico al integrar los diferentes sistemas de gestión de la empresa y, evidentemente, pueden favorecer el trabajo en equipo y la comunicación a través de herramientas como las intranets, el correo electrónico y las aplicaciones de apoyo al trabajo en grupo y a las comunidades de práctica.

Por último, la composición del equipo directivo es otro factor crítico que puede ser intervenido para favorecer el aprendizaje. Claramente, el liderazgo facilitador del aprendizaje es un atributo que solo se desarrolla cuando el equipo directivo está conformado por personas que practican dicho estilo de liderazgo y se convierten en referentes para los demás miembros de la organización. No es posible crear una cultura de aprendizaje si los líderes no son consecuentes con dicha cultura.

Este artículo presenta las limitaciones propias de una revisión hermenéutica de la literatura. A pesar de la profundidad de interpretación que permite este enfoque, no se puede desconocer el nivel de subjetividad implícito al sin- 
tetizar y articular los elementos más relevantes de modelos teóricos diversos. Adicionalmente, el carácter prescriptivo de varios de los modelos teóricos analizados ha influido en que varias de las conclusiones conserven dicho carácter prescriptivo.

Teniendo en cuenta las críticas que se plantearon a dicho enfoque de que está más orientado a los profesionales que a la comunidad académica, es importante desarrollar futuras investigaciones que brinden un mayor rigor y respaldo empírico a dichas conclusiones.

Futuras investigaciones pueden ayudar a probar empíricamente la influencia de estas decisiones asociadas a la estrategia, la estructura, las políticas de gestión humana, las tecnologías de la información y la composición del equipo directivo sobre los atributos de la organización que aprende. De igual forma, puede ser valioso investigar la relación entre los atributos identificados en este trabajo y algunas dimensiones del desempeño organizacional, tales como los resultados de innovación, la rentabilidad, el crecimiento y la sostenibilidad de la ventaja competitiva.

Otra línea de investigación que puede desprenderse de este trabajo consiste en analizar las relaciones entre los atributos de la organización que aprende y cada uno de los procesos específicos del aprendizaje: la adquisición, la distribución, la interpretación del conocimiento y la memoria organizativa.

Finalmente, podría investigarse a través de futuros estudios el impacto diferenciado de cada atributo de la organización que aprende sobre los tipos específicos de aprendizaje de exploración y de explotación, o sobre la capacidad de las empresas para desarrollar ambos tipos de aprendizaje de forma simultánea a través de la ambidestreza organizativa.

\section{Referencias bibliográficas}

Alavi, M., \& Leidner, D. E. (2001). "Review: Knowledge management and knowledge management systems: conceptual foundations and research issues". MIS Quarterly, 25(1), 107-136.

Argyris, C., \& Schön, D. A. (1978). Organizational learning: A theory of action perspective. MA: Addison-Wesley Reading.

BARNEY, J. (1991). "Firm resources and the theory of competitive advantage". Journal of management, 17(1), 99-120.

Bass, B. M., \& Riggio, R. E. (2006). Transformational leadership. New Jersey: Lawrence Erlbaum Associates. 
Bontis, N. (1998). "Intellectual capital: an exploratory study that develops measures and models". Management Decision, 36(2), 63-76.

Bontis, N.; Crossan, M. M., \& Hulland, J. (2002). "Managing an Organizational Learning System by aligning stocks and flows". Journal of Management studies, 39(4), 437-469.

Cangelosi, V.E., \& Dill, W.R.(1965).“'Organizational learning: Observations toward a theory". Administrative Science Quarterly, 10(2), 175-203.

Cardona, J. A., \& Calderón, G. (2006). “El impacto del aprendizaje en el rendimiento de las organizaciones". Cuadernos de Administración, 19(32), $11-43$.

Cohen, W. M., \& Levinthal, D. A. (1990). "Absorptive Capacity: A New Perspective on Learning and Innovation". Administrative Science Quarterly, 35(1), 128-152.

Crossan, M. M.; Lane, H. W., \& White, R. E. (1999). “An Organizational Learning Framework: From Intuition to Institution". Academy of Management Review, 24(3), 522-537.

Cyert, R. M., \& March, J. G. (1963). A Behavioral Theory of the Firm. Englewoods Cliffs, N.Y.: Prentice-Hall.

Chen, G. (2005). "An organizational learning model based on western and Chinese management thoughts and practices". Management Decision, 43(4), 479-500.

Chiva, R.; Alegre, J., \& Lapiedra, R. (2007). “Measuring organisational learning capability among the workforce". International Journal of Manpower, 28(3/4), 224-242.

Duncan, R. B., \& Weiss, A. (1979). “Organizational learning: Implications for organizational design”. En B. STAw (Ed.), Research in Organizational Behavior (Vol. 1, pp. 75-123). Greenwich: JAI Press.

Easterby-Smith, M. (1997). "Disciplines of organizational learning: contributions and critiques". Human Relations, 50(9), 1085-1113.

Easterby-Smith, M.; Crossan, M., \& Nicolini, D. (2000). “Organizational learning: debates past, present and future". Journal of Management studies, 37(6), 783-796.

Fiol, C. M., \& Lyles, M. A. (1985). "Organizational Learning”. Academy of Management Review, 10(4), 803-813.

Forrester, J. W. (1971). "Counterintuitive Behavior of Social Systems". Technology Review, 73(3), 52-68.

Garvin, D. A. (1993). "Building a learning organization". Harvard Business Review, 71(4), 78-91. 
Garvin, D. A.; Edmondson, A. C., \& Gino, F. (2008). "Is yours a learning organization?” Harvard Business Review, 86(3), 109-116.

Goh, S., \& Richards, G. (1997). "Benchmarking the learning capability of organizations". European Management Journal, 15(5), 575-583.

Gupta, A. K., \& Govindarajan, V. (1991)."Knowledge flows and the structure of control within multinational corporations". Academy of Management Review, 16(4), 768-792.

Hedberg, B. (1981). "How organizations learn and unlearn?” En P. C. Nystrom \& W. H. Starbuck (Eds.), Handbook of organizational design (Vol. 1, pp. 3-27). New York: Oxford University Press.

Huber, G. P. (1991). "Organizational Learning* The Contributing Processes and the Literatures". Organization science, 2(1), 88-115.

Hult, G. T. M., \& Ferrell, O. C. (1997). "Global organizational learning capacity in purchasing: construct and measurement". Journal of business research, 40(2), 97-111.

Jerez, P. (2001). "La gestión de recursos humanos y el aprendizaje organizativo: Incidencia e implicaciones”. Almería: Tesis Doctoral, Universidad de Almería. Jerez, P.; Céspedes, J., \& VAlle, R. (2005). “Organizational learning capability* a proposal of measurement". Journal of business research, 58(6), 715-725.

Kiм, D. H. (1993). "The Link Between Individual and Organizational Learning”. Sloan Management Review, 35(1), 37-50.

Kogut, B., \& Zander, U. (1992). "Knowledge of the Firm, Combinative Capabilities, and the Replication of Technology". Organization science, 3(3), 383-397.

Levit', B., \& March, J. G. (1988). “Organizational Learning”. Annual Review of Sociology, 14, 319-340.

López-Zapata, E*; García-Muiña, F. E., \& García-Moreno, S. M. (2012). "De la organización que aprende a la organización ambidiestra: evolución teórica del aprendizaje organizativo". Cuadernos de Administración, 25(45), $11-37$.

Lyles, M. A. (2014). “Organizational Learning, knowledge creation, problem formulation and innovation in messy problems". European Management Journal, 32(1), 132-136.

Lloria, M. B., \& Moreno-Luzon, M. D. (2014). “Organizational learning; Proposal of an integrative scale and research instrument". Journal of business research, 67(5), 692-697.

March, J. G. (1991). "Exploration and Exploitation in Organizational Learning”. Organization science, 2(1), 71-87. 
March, J. G., \& Olsen, J. P. (1975). “The uncertainty of the past: Organizational learning under ambiguity". European Journal of Political Research, 3(2), 147 171.

McGill, M. E*; Slocum, J. W., \& Lei, D. (1992). "Management practices in learning organizations". Organizational dynamics, 21(1), 5-17.

Mintzberg, H. (1988). La estructuración de las organizaciones. Barcelona: Ariel Economía.

Nelson, R. R., \& Winter, S. G. (1982). An Evolutionary Theory of Economic Change. Cambridge, MA: Harvard University Press.

Nevis, E. C.; Dibella, A. J., \& Gould, J. M. (1995). "Undestanding organizations as learning systems". Sloan Management Review, 36(2), 73-85.

Nonaka, I., \& Takeuchi, H. (1995). The knowledge-creating company: how japanese companies create the dynamics of innovation. New York: Oxford University Press.

Nonaka, I., \& Von Krogh, G. (2009). "Tacit knowledge and knowledge conversion: Controversy and advancement in organizational knowledge creation theory". Organization science, 20(3), 635-652.

Raisch, S., \& Birkinshaw, J. (2008). "Organizational ambidexterity: Antecedents, outcomes, and moderators". Journal of management, 34(3), 375409.

Senge, P. M. (1990). The fifth discipline: The art and practice of the learning organization. New York: Doubleday.

Shipton, H. (2006). "Cohesion or confusion? Towards a typology for organizational learning research". International Journal of Management Reviews, 8(4), 233-252.

Shipton, H.; Zhou, Q., \& Moor, E. (2013)."Is there a global model of learning organizations? An empirical, cross-nation study”. International Journal of Human Resource Management, 24(12), 2278-2298.

Shrivastava, P. (1983). “A typology of organizational learning systems”. Journal of Management studies, 20(1), 7-28.

Sinkula, J. M.; Baker, W. E., \& Noordewier, T. (1997). A framework for market-based organizational learning: linking values, knowledge, and behavior. Journal of the Academy of Marketing Science, 25(4), 305-318.

Slater, S. F., \& Narver, J. C. (1995). "Market orientation and the learning organization". Journal of Marketing, 59(3), 63-74.

Slocum, J. W*; McGill, M. E., \& Lei, D. T. (1994)."The new learning strategy* Anytime, anything, anywhere". Organizational dynamics, 23(2), 33-47. 
Stata, R. (1989).“Organizational Learningः the key to management innovation”. Sloan Management Review, 30(3), 63-74.

Teece, D. J. (2009). Dynamic Capabilities and Strategic Management: Organizing for Innovation and Growth. New York: Oxford University Press.

Teece, D. J; Pisano, G.; \& Shuen, A. (1997). "Dynamic Capabilities and Strategic Management". Strategic management journal, 18(7), 509-533.

Templeton, G. F.; Lewis, B. R., \& Snyder, C. A. (2002). "Development of a measure for the organizational learning construct". Journal of Management Information Systems, 19(2), 175-218.

Tsang, E. W. K. (1997)." Organizational learning and the learning organization: a dichotomy between descriptive and prescriptive research". Human Relations, 50(1), 73-89.

Vera, D; Crossan, M., \& Apaydin, M. (2011)."A framework for integrating organizational learning, knowledge, capabilities, and absorptive capacity". En M. Easterby-Smith \& M. A. Lyles (Eds.), Handbook of organizational learning and knowledge management (2 ed., pp. 153-180). Chichester: Wiley.

Walsh, J. P., \& Ungson, G. R. (1991). “Organizational memory”. Academy of Management Review, 16(1), 57-91.

Wang, C. L., \& RafiQ, M. (2009)."Organizational diversity and shared vision: Resolving the paradox of exploratory and exploitative learning". European Journal of Innovation Management, 12(1), 86-101.

Yang, B.; Watkins, K. E., \& Marsick, V. J. (2004). "The construct of the learning organization: Dimensions, measurement, and validation". Human Resource Development Quarterly, 15(1), 31-55.

Zноu, W; Hu, H., \& SHI, X. (2015). "Does organizational learning lead to higher firm performance? An investigation of Chinese listing companies". The Learning Organization, 22(5), 271-288.

Zollo, M., \& Winter, S. G. (2002). "Deliberate Learning and the Evolution of Dynamic Capabilities". Organization science, 13(3), 339-351. 